\title{
Evaluation of Binding of Perylene Diimide and Benzannulated Perylene Diimide Ligands to DNA by Electrospray Ionization Mass Spectrometry
}

\author{
Carolyn L. Mazzitelli and Jennifer S. Brodbelt \\ Department of Chemistry and Biochemistry, The University of Texas at Austin, Austin, Texas, USA
}

\author{
Jonathan T. Kern, Mireya Rodriguez, and Sean M. Kerwin \\ Division of Medicinal Chemistry and Institute for Cellular and Molecular Biology, The University of Texas at \\ Austin, Austin, Texas, USA
}

\begin{abstract}
Electrospray ionization mass spectrometry (ESI-MS) and spectroscopic studies in solution were used to evaluate the self-association, G-quadruplex DNA binding, and selectivity of a series of perylene diimides (PDIs) (PIPER, Tel01, Tel11, Tel12, and Tel18) or benzannulated perylene diimide ligands (Tel34 and Tel32). Fluorescence and resonance light scattering spectra of Tel01, Tel12, Tel32, and Tel34 reveal that these analogs undergo self-association in solution. UV-Vis and fluorescence titrations with G-quadruplex, duplex, or single-stranded DNA demonstrate that all the analogs, with the exception of Tel32, bind to G-quadruplex DNA, with those PDIs that are self-associated in solution showing the highest degree of selectivity for binding G-quadruplex DNA. Parallel ESI-MS analysis of the stoichiometries demonstrates the ability of the ligands, with the exception of Tel32, to bind to G-quadruplex DNA. While most ligands show major 1:1 and 2:1 binding stoichiometries as expected in the case of end-stacking, interestingly, three of the most quadruplex-selective ligands show a different behavior. Tel01 forms 3:1 complexes, while Tel12 and Tel32 only form 1:1 complexes. Collisional activation dissociation patterns are compatible with ligand binding to G-quadruplex DNA via stacking on the ends of the terminal G-tetrads. Experiments with duplex and single strand DNA were performed to assess the binding selectivities of the ligands. PIPER, Tel11, and Tel18 demonstrated extensive complexation with duplex DNA, while Tel11 and Tel18 bound to single strand DNA, confirming the lack of selectivity of these two ligands. Our results indicate that Tel01, Tel12, and Tel34 are the most selective for G-quadruplex DNA. (J Am Soc Mass Spectrom 2006, 17, 593-604) (c) 2006 American Society for Mass Spectrometry
\end{abstract}

S mall molecules that interact noncovalently with nucleic acid structures comprise an important class of anticancer, antitumor, and antibacterial therapies [1,2]. Increased interest in the development and evaluation of DNA-interactive agents has stimulated the need for sensitive analytical techniques that can not only characterize drug/DNA interactions but are also compatible with library-based screening. Electrospray ionization-mass spectrometry (ESI-MS) has emerged as a useful tool for examining noncovalent drug/DNA complexes because its low sample consumption and fast analysis time makes it well-suited for high throughput screening techniques [3,4]. The fullscan mass spectra can be used to evaluate binding

Published online February 28, 2006

Address reprint requests to Dr. J. S. Brodbelt, Department of Chemistry and Biochemistry, University of Texas at Austin. 1 University Station A5300, Austin, TX 78712-0165, USA. E-mail: jbrodbelt@mail.utexas.edu stoichiometries and selectivity, while binding mode and structural information can be examined via tandem mass spectrometry techniques such as collisional activated dissociation (CAD) [5-7].

Much of the past work done in this area has focused on analyzing well-characterized drug/duplex DNA complexes, with promising results that indicate behavior in the gas phase can be correlated to solution [5-25]. For example, Gabelica and coworkers demonstrated that binding stoichiometries and relative ion abundances observed in the mass spectra reflect known solution binding behavior [6]. Minor groove and intercalation binding modes of well-studied duplex-interactive drugs were distinguished by Wan and coworkers using collisional activated dissociation (CAD) experiments [7].

Recent work by our group and others has extended the use of ESI-MS to evaluate noncovalent interactions of small molecules with G-quadruplex DNA [18, 20, 23, 

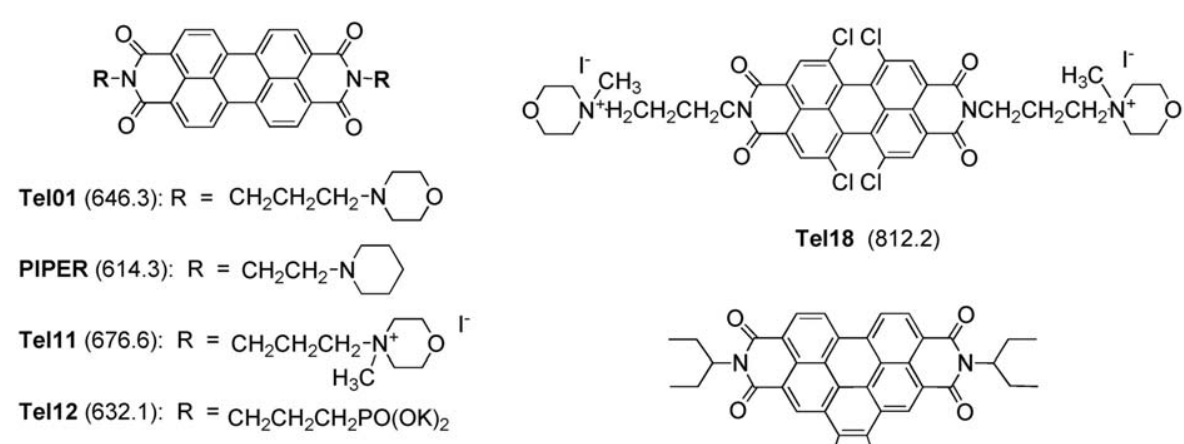

Tel18 (812.2)

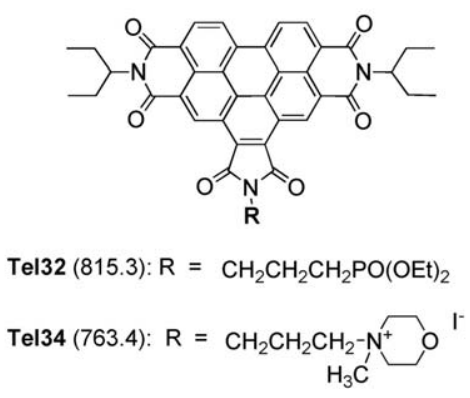

Figure 1. Structures of perylene diimide analogs. Molecular weights of compounds (Tel01, PIPER, Tel32) or organic ions (Tel11, Tel12, Tel18, Tel34) in Da are given in parenthesis.

24, 26-28], a higher order nucleic acid structure being investigated as an anticancer drug target $[29,30]$. Gquadruplex DNA is formed from hydrogen bonding between a planar arrangement of four guanine nucleobases with the central cavity serving as a binding site for monovalent metal ions, the presence of which is generally required for the formation of the quadruplex. A variety of G-quadruplex structures can be formed from hydrogen bonding between guanines on up to four separate strands of oligodeoxynucleotides (ODNs) [29].

There is interest in studying G-quadruplex DNA as an anticancer target because of its role in telomere maintenance in cancer cells [31, 32]. Telomeres are the extremities of linear chromosomes that play an important role in protecting the chromosome ends from fusion and degradation and ensuring the complete replication of chromosomal DNA. Composed of G-rich sequences (TTAGGG is the human telomeric sequence), the single strand $3^{\prime}$ overhangs of telomeres have the ability to form G-quadruplex structures. Ligands that selectively bind and stabilize the quadruplex structure of telomeric DNA may lead to the inhibition of telomerase, the enzyme responsible for the synthesis of telomeric DNA [33]. While telomerase is not active in most somatic cells, high levels of telomerase activity have been associated with most cancer cells [34]. Developing a drug that exhibits binding selectivity for Gquadruplex DNA over other DNA structures is also a primary focus of G-quadruplex-interactive drug design, given the implications for reducing cytotoxic sideeffects of anticancer agents.

One promising class of quadruplex selective ligands are perylene diimides (PDIs) [35-43]. Recent solution studies of $N, N^{\prime}$-bis[2-(1-piperidino)-ethyl]-3,4,9,10perylenetetracarboxylic acid diimide (PIPER) [35] and N, N'-bis(4-morpholinylpropyl)-3,4,9,10-perylenetetracarboxylic acid diimide (Tel01) [38] indicate that these molecules exhibit G-quadruplex affinity by stacking on the faces of the terminal G-tetrads, thereby stabilizing the G-quadruplex structure. We previously demonstrated the selectivity of Tel01 for quadruplex over duplex DNA based on ESI-MS studies in a quadrupole ion trap mass spectrometer [26]. A more comprehensive series of PDI ligands, including those in which the perylene diimide chromophore is modified through substitution or benzannulation (Figure 1), have been designed to further investigate the structural features most important for G-quadruplex DNA binding. In this study we demonstrate the utility of ESI-MS as a screening tool to characterize binding stoichiometry, distinguish between selective and nonselective binding, and determine the binding mode of these novel perylene diimide ligands.

\section{Experimental Procedures}

\section{Chemicals}

PIPER, Tel01, Tel11, Tel12 were prepared as described previously $[35,37,38]$. The manuscript detailing the synthesis of Tel18, Tel32, and Tel34 is in preparation. Ammonium salts of oligodeoxynucleotides (ODNs) custom synthesized on the $1.0 \mu$ mole scale with purification by RP-HPLC were obtained from TriLink Biotechnologies Inc. (San Diego, CA) and Integrated DNA Technologies (Coralville, IA) and used without further purification. The oligonucleotide d(TTTTTTTT) was synthesized on a $10 \mu \mathrm{M}$ scale using a DNA synthesizer and purified by RP-HPLC. Collected fractions were combined and extensively dialyzed against deionized water before being lyophilized completely. A $2 \mathrm{mM}$ stock solution of the G-quadruplex-forming ODN (d(TTGGGGGT)) was prepared in deionized water. A portion of the initial stock solution was diluted to $500 \mu \mathrm{M}$ in deionized water and set aside for single strand experiments. The remaining solution was annealed 
by diluting in $150 \mathrm{mM}$ ammonium acetate, heating to $90^{\circ} \mathrm{C}$ and slowly cooling to room temperature over a period of $7 \mathrm{~h}$. The self-complementary duplex-forming ODNs (d(GCGGGGATGGGGCG/ CGCCCCATCCCCGC), d(GCGGGAATTGGGCG/ CGCCCAATTCCCGC), and d(GCGGAAATTTGGCG/ CGCCAAATTTCCGC)) were annealed in $250 \mathrm{mM}$ ammonium acetate by heating to $90^{\circ} \mathrm{C}$ and slowly cooling to room temperature over a period of $2-3 \mathrm{~h}$.

\section{Absorption Spectroscopy}

Spectra were recorded on a UNICO model 2102 UVspectrophotometer (Dayton, NJ). Experiments were carried out in polystyrene cuvettes to minimize nonspecific binding of the ligands to the surface of the cuvettes. For DNA binding experiments, the absorption spectra were obtained under high and low salt conditions. For the high salt conditions, the compound $(20 \mu \mathrm{M})$ in $70 \mathrm{mM}$ potassium phosphate, $100 \mathrm{mM}$ potassium chloride, 1 mM EDTA buffer ( $\mathrm{pH} \mathrm{7)}$ was analyzed alone or in the presence $20 \mu \mathrm{M}$ structure of G4-DNA [d(TAGGGTTA) $]_{4}$, G4'-DNA [d(TTAGGG) ${ }_{4}$ ] doublestrand DNA [d(CGCGCGATATCGCGCG) $]_{2}$, or singlestranded DNA d(TTTTTTTT). For the low salt conditions, the compound $(10 \mu \mathrm{M})$ in 3:1 $25 \mathrm{mM}$ ammonium acetate/methanol was analyzed alone or in the presence of $10 \mu \mathrm{M}$ structure of G4-DNA [d(TT-

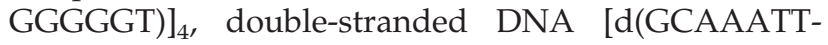
TCG) $]_{2}$ or single-stranded DNA d(TTTTTTTT). Samples were monitored until equilibrium was achieved, as evidenced by constant absorbance readings.

\section{Fluorescence Spectroscopy and Resonance Light Scattering}

Spectra were recorded on a Hitachi model F-2000 spectrofluorometer. Quartz cuvettes were treated with SigmaCote (Sigma Aldrich, Milwaukee, WI) for $1 \mathrm{~h}$ followed by extensive washing with water to minimize nonspecific binding of the ligands to the surface of the cuvette. Fluorescence and resonance light scattering (RLS) measurements were carried out on the ligands under both high- and low-salt conditions. The high-salt conditions employed solutions of compound in $70 \mathrm{mM}$ potassium phosphate, $100 \mathrm{mM}$ potassium chloride, 1 mM EDTA buffer at the indicated $\mathrm{pH}$. Low-salt conditions employed solutions of compound in 3:1 $25 \mathrm{mM}$ ammonium acetate/methanol at the indicated $\mathrm{pH}$. Solutions were allowed to equilibrate for the specified time before scans were performed at $25^{\circ} \mathrm{C}$ using the noted excitation and emission wavelengths.

\section{Mass Spectrometry}

Stock solutions of the ligands were prepared in either $0.1 \%$ aqueous trifluoroacetic acid (PIPER and Tel01), deionized water (Tel11, Tel12, and Tel18), acetonitrile
(Tel32) or dimethylsulfoxide (Tel34) and stored at room temperature. Analytical solutions were prepared containing G-quadruplex, duplex, or single strand DNA and one ligand each at equimolar $10 \mu \mathrm{M}$ concentrations in 3:1 $25 \mathrm{mM}$ ammonium acetate/methanol solvent. A Harvard syringe pump (Holliston, MA) set at a flow rate of $3 \mu \mathrm{L} / \mathrm{min}$ was used to directly infuse the sample solutions into a ThermoFinnigan LCQ Duo mass spectrometer (San Jose, CA). The ESI source was operated in the negative ion mode with an electrospray voltage of $3.5 \mathrm{kV}$ and a heated capillary temperature of 90 to $120^{\circ} \mathrm{C}$. To aid in desolvation, nitrogen sheath and auxiliary gas were set at 40 and 10 arbitrary units, respectively. The base pressure of the trap was $\sim 1 \times$ $10^{-5}$ torr. Spectra were acquired by summing 300 scans, with an ionization time ranging from 100 to $250 \mathrm{~ms}$.

Collisional activated dissociation (CAD) experiments were performed on selected complexes by isolating the desired precursor ion in the ion trap using resonance ejection, followed by fragmentation promoted by increasing the collisional energy applied to the trap (reported as a percentage of $5 \mathrm{~V}_{0-p}$ ) until the intensity of the precursor ion was reduced to approximated $10 \%$ of its original intensity. An activation time of $30 \mathrm{~ms}$ was used in all CAD experiments.

\section{Results and Discussion}

\section{Fluorescence and Resonance Light Scattering Studies of PDI Aggregation}

PDIs like Tel01 and PIPER are known to undergo $\mathrm{pH}$-dependent self-association to form aggregates in solution [37-39]. The selectivity of PDIs towards Gquadruplex DNA depends on the aggregation state of these ligands, which, in addition to $\mathrm{pH}$, may also be affected by ligand concentration, buffer ionic strength, and the presence of nonaqueous cosolvents [36, 38]. Previous solution studies of the aggregation state and G-quadruplex DNA binding selectivity of PIPER [36, 38], Tel01 [36, 38], Tel11 [37], and Tel12 [37] were carried out under relatively high-salt $(170 \mathrm{mM} \mathrm{KCl})$ phosphate buffer. To determine the aggregation state of these and the other PDI ligands under the conditions of the ESI-MS analysis discussed later, the fluorescence and resonance light scattering spectra were determined in 3:1 $25 \mathrm{mM}$ ammonium acetate/methanol.

As reported previously, the intense fluorescence of the monomeric PDIs is quenched upon ligand selfassociation in solution [36-38]. Solutions of PIPER (1 $\mu \mathrm{M}$ ) in methanolic ammonium acetate buffer display a pronounced fluorescence emission at $550 \mathrm{~nm}$ whereas solutions of Tel01 do not (Supplementary Figure S-1 which can be found in the electronic version of this article.). For aggregates in which there is good overlap between adjacent monomer chromophores, a large increase in the intensity of scattered light is observed when the incident light is the same wavelength as the aggregate absorbance [44]. This resonance light scatter- 
Table 1. Resonance light scattering and fluorescence data for PDIs

\begin{tabular}{lcc}
\hline Ligand & $\begin{array}{c}\text { Relative intrinsic } \\
\text { fluorescence }^{\mathrm{a}}\end{array}$ & $\begin{array}{c}\text { Relative RLS } \\
\text { signal }^{\mathrm{b}}\end{array}$ \\
\hline \hline TEL01 & 0.649 & 36 \\
PIPER & 25.31 & $\mathrm{~ns}^{\mathrm{c}}$ \\
TEL11 & 25.83 & $\mathrm{~ns}^{\mathrm{c}}$ \\
TEL12 & 12.74 & $\mathrm{~ns}^{\mathrm{c}}$ \\
TEL18 & 41.82 & $\mathrm{~ns}^{\mathrm{c}}$ \\
TEL32 & 0.189 & 6 \\
TEL34 & 0.524 & 5 \\
\hline
\end{tabular}

Relative intensity of fluorescence emission (550 nm) from a $1 \mu \mathrm{M}$ solution of PDI in 3:1 $25 \mathrm{mM}$ ammonium acetate/methanol buffer, $\mathrm{pH} 7$. Excitation was at $520 \mathrm{~nm}$ for all ligands except Tel32 and Tel24, which were excited at $495 \mathrm{~nm}$.

${ }^{b}$ Relative intensity of the resonance light scattering peak at $470 \mathrm{~nm}$ for solutions of PDI (1 $\mu \mathrm{M})$ in $3: 125 \mathrm{mM}$ ammonium acetate/methanol buffer, $\mathrm{pH} 7$.

${ }^{\mathrm{c}}$ No significant RLS signal observed.

ing (RLS) signal has been used as a qualitative measure of the presence of PDI aggregates in solution [36-38]. Solutions of Tel01 display a large RLS signal; however, the RLS signal for PIPER is absent, as demonstrated in Figure S-1. The absence of strong fluorescence and the presence of a strong RLS signal for solutions of Tel01 indicate that this PDI is aggregated under these conditions. In contrast, the lack of an RLS signal and the presence of strong fluorescence for solutions of PIPER indicate that this PDI exists in the monomeric state. Similar observations were made for solution of Tel01 and PIPER in high-salt buffer conditions, indicating that the aggregation state of these two PDIs is largely unaffected by the methanolic buffer conditions employed in the ESI-MS analysis.

The fluorescence and RLS spectra of the other PDIs were also examined under the methanolic buffer conditions. As shown in Table 1, PIPER, Tel11, and Tel18 are not aggregated in solution, as judged by the strong fluorescence and lack of RLS signals of these solutions. The PDI Tel12 displays only weak fluorescence and no RLS spectra. The weak fluorescence of Tel12 may be due to ligand dimerization, as has been noted for this PDI in $150 \mathrm{mM} \mathrm{KCl}$ phosphate buffer [37]; however, the fluorescence intensity of Tel12 in methanolic buffer is higher than that in the high-salt buffer (data not shown), indicating that dimerization is less extensive in the methanolic buffer. Because Tel12 has been found to dimerize, but does not produce a RLS spectrum, this ligand self-associates. Self-association refers to the formation of dimers, as is the case for Tel12, as well as high-order species and aggregates, which we functionally define as PDI species that can be observed by resonance-light scattering.

Like Tel01, solutions of the benzannulated PDI Tel34 in methanolic buffer do not fluoresce; however, unlike Tel01, Tel34 solutions do not display a strong RLS signal, indicating that if Tel34 is aggregated in solution, the aggregates are RLS-silent. Attempts to compare the aggregation state of Tel34 in methanolic ammonium acetate and $150 \mathrm{mM} \mathrm{KCl}$ phosphate buffer were unsuccessful due to the very low solubility of this PDI in the high-salt buffer. Similarly, the low solubility of Tel32, even in methanolic ammonium acetate, made characterization of this PDI difficult. Immediately prepared solutions of Tel32 showed very low fluorescence and RLS signals; however, over a period of minutes the ligand began to precipitate from these solutions.

\section{DNA Binding Studies by UV-Vis Absorption Spectroscopy}

The UV-Vis absorption spectra of the PDIs (10 $\mu \mathrm{M}$ in $3: 1$ $25 \mathrm{mM}$ ammonium acetate/methanol) were determined in the absence and presence of $10 \mu \mathrm{M}$ G-quadruplex DNA, duplex DNA, or single-stranded DNA. The parallel stranded G-quadruplex $\left[\mathrm{d}\left(\mathrm{T}_{2} \mathrm{G}_{5} \mathrm{~T}\right)\right]_{4}(\mathrm{G} 5)$, which is similar to the well-characterized $\left[\mathrm{d}_{2}\left(\mathrm{~T}_{2} \mathrm{G}_{4} \mathrm{~T}\right)\right]_{4}$, was employed for both these solution binding studies as well as the ESI-MS analysis. An advantage of this parallelstranded G-quadruplex over intramolecular G-quadruplexes is the ease with which the G-quadruplex form can be distinguished from single-stranded DNA in the ESI-MS spectra (see below). The duplex DNA formed by the self-complementary ODN d(GCAAATTTCG) and single-stranded $\mathrm{d}\left(\mathrm{T}_{8}\right)$ were also used in these solution binding studies.

As shown in Figure 2a, the UV-Vis spectrum of Tel01 shows an absorbance peak at $470 \mathrm{~nm}$. Similar absorbance spectra are recorded for solutions of Tel01 in the presence of equimolar duplex or single-stranded DNA; however, in the presence of equimolar G-quadruplex DNA, the absorbance spectrum of Tel01 is remarkably different, consisting of two large peaks at 550 and 510 $\mathrm{nm}$. The UV-Vis spectrum of PIPER displays an absorbance peak at $500 \mathrm{~nm}$, which shifts to $510 \mathrm{~nm}$ in the presence of equimolar single-stranded, doublestranded, or G-quadruplex DNA (Figure 2b). Additionally, in the presence of each of these DNA structures, there is a new absorbance from the PIPER chromophore at $550 \mathrm{~nm}$. The intensity of this long-wavelength band increases in the order single-stranded DNA $<$ doublestranded DNA $<$ G-quadruplex DNA.

These UV-Vis spectral changes for Tel01 and PIPER indicate a difference in the G-quadruplex DNA binding selectivity of these two ligands [37, 38]. Tel01, whose absorbance spectrum in the absence of DNA is blueshifted due to ligand aggregation, forms a complex with G-quadruplex DNA that absorbs in the $550 \mathrm{~nm}$ region. PIPER, whose spectrum in the absence of DNA does not indicate ligand aggregation, forms similar long-wavelength absorbing complexes with G-quadruplex, duplex, and even single-stranded DNA (Figure 2b).

Other PDIs shown in Figure 1 also undergo UV-Vis spectral changes in the presence of G-quadruplex DNA. Tel11 and Tel12, whose absorbance spectra in the absence of DNA is similar to that of PIPER, also form complexes with G-quadruplex DNA characterized by 

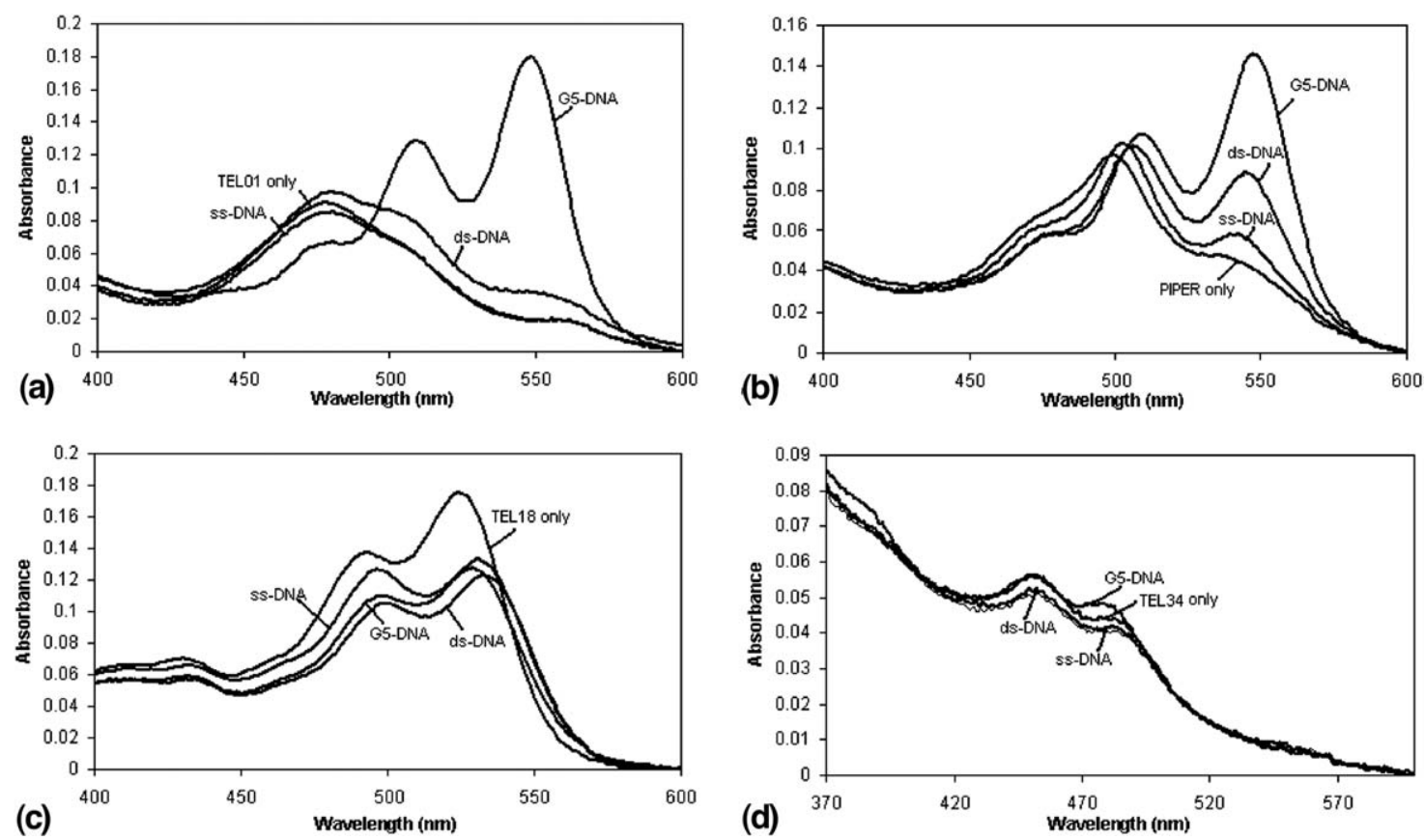

Figure 2. UV-Vis absorbance spectra of $10 \mu \mathrm{M}$ Tel01(a), PIPER (b), Tel18 (c), and Tel34 (d) alone and in the presence of $10 \mu \mathrm{M}$ G5-DNA $\left[\mathrm{d}\left(\mathrm{T}_{2} \mathrm{GT}\right)\right]_{4}$, ds-DNA [d(GCAAATTTCG) $]_{2}$, or ss-DNA [d( $\left.\left.\mathrm{T}_{8}\right)\right]$ in 3:1 $25 \mathrm{mM}$ ammonium acetate/methanol, $\mathrm{pH} 7$.

absorbance peaks at $550 \mathrm{~nm}$ (data not shown). Tel11, but not Tel12, also gives rise to a peak at $550 \mathrm{~nm}$ in the presence of double-stranded DNA. As expected, the UV-Vis absorbance spectrum of the chromophoremodified PDI Tel18 is different from that of PIPER and the other PDIs. The Tel18 spectrum, consisting of a peak at $525 \mathrm{~nm}$ with a shoulder at $492 \mathrm{~nm}$, undergoes hypochromic and bathochromic shifts in the presence of G-quadruplex DNA (Figure 2c). Similar changes are also observed in the presence of duplex DNA, and to a lesser extent, single-stranded DNA. The absorbance spectrum of Tel34 includes a long wavelength peak at $450 \mathrm{~nm}$ with a shoulder at $490 \mathrm{~nm}$ (Figure 2d). In the presence of G-quadruplex DNA, there is a shift of the $490 \mathrm{~nm}$ absorbance to $480 \mathrm{~nm}$, accompanied by slight hyperchromism. Immediately after the addition of double-stranded or single-stranded DNA, there is a slight hypochromism in the Tel34 absorbance spectrum, but no appreciable changes in the position of the absorbance peaks (Figure 2d). Over a period of an hour, solutions of Tel34 containing duplex or single-stranded DNA exhibit a pronounced decrease in UV-Vis absorbance that is accompanied by the formation of insoluble material. This behavior is not observed in the presence of G5 DNA, presumably because the complex formed between this benzannulated PDI and the G-quadruplex DNA is more soluble in the buffer than the ligand itself. The benzannulated and uncharged PDI Tel32 is also relatively insoluble, and at the concentration employed for these absorbance studies $(10 \mu \mathrm{M})$, the ligand precipitated from solution over the course of a few minutes.

\section{Fluorescence Quenching Studies}

It has previously been shown that PDI fluorescence is quenched upon ligand binding to DNA structures [3638]. Fluorescence-quenching experiments of the PDIs with quadruplex, duplex, and single-stranded DNA allow better quantification and insight into the affinity and selectivity differences of these ligands for G-quadruplex DNA. Solutions of $1 \mu \mathrm{M}$ PDI in 3:1 $25 \mathrm{mM}$ ammonium acetate/ methanol buffer, $\mathrm{pH} 7$ were titrated with stock solutions of each DNA structure in the same buffer. Fluorescence spectra were recorded at appropriate emission and excitation wavelengths for each class of chromophore: unmodified PDIs Tel01, Tel11, Tel12, Tel34, and PIPER (emission at $545 \mathrm{~nm}$, excitation at $495 \mathrm{~nm}$ ); chlorinated PDI Tel18 (emission at $555 \mathrm{~nm}$, excitation at $495 \mathrm{~nm}$ ); and benzannulated PDI Tel32 (emission at $565 \mathrm{~nm}$, excitation at $469 \mathrm{~nm}$ ). (Examples of the fluorescence titration curves for Tel18 with quadruplex G5-DNA [d(TTGGGGGT) $]_{4}$, duplex $[\mathrm{d}(\mathrm{GCAAATTTCG})]_{2}$, and single-stranded $\left[\mathrm{d}\left(\mathrm{T}_{8}\right)\right]$ are shown in Supplementary Figure S-2 which can be found in the electronice version of this article.).While all three DNA samples quench the fluorescence of Tel18, there is clearly a difference between the efficiency of fluorescence-quenching, with G5-DNA causing the most extensive quenching at low concentrations, singlestranded DNA producing the least quenching, and duplex DNA leading to intermediate quenching. In the case of G-quadruplex DNA, there is nearly $80 \%$ fluorescencequenching after the addition of just one-half equivalent of DNA, indicating that multiple Tel18 ligands are binding to the DNA. 
Table 2. Fluorescence quenching of PDIs by quadruplex, duplex, and single-stranded DNA

\begin{tabular}{lccr}
\hline & \multicolumn{2}{c}{$\begin{array}{c}\text { Percent fluorescence quenching in the } \\
\text { presence of equimolar DNA }\end{array}$} \\
\cline { 2 - 4 } Ligand & G5-DNA $^{\mathrm{b}}$ & \multicolumn{1}{c}{ ds-DNA $^{\mathrm{c}}$} & ss-DNA $^{\mathrm{d}}$ \\
\hline \hline TEL01 $^{\mathrm{e}}$ & $64 \pm 4 \%$ & $6 \pm 2 \%$ & $2 \pm 1 \%$ \\
PIPER $^{\mathrm{e}}$ & $100 \pm 1 \%$ & $94 \pm 2 \%$ & $57 \pm 4 \%$ \\
TEL11 $^{\mathrm{e}}$ & $99.8 \pm 0.1 \%$ & $96 \pm 1 \%$ & $67 \pm 5 \%$ \\
TEL12 $^{\mathrm{e}}$ & $29 \pm 6 \%$ & $16 \pm 8 \%$ & $13 \pm 6 \%$ \\
TEL18 $^{\mathrm{f}}$ & $92.9 \pm 0.1 \%$ & $66 \pm 3 \%$ & $12 \pm 9 \%$ \\
TEL32 $^{\mathrm{c}}$ & $4 \pm 2 \%$ & $1 \pm 2 \%$ & $7 \pm 2 \%$ \\
TEL34 $^{\mathrm{g}}$ & $90 \pm 2 \%$ & $62 \pm 4 \%$ & $40 \pm 6 \%$ \\
\hline
\end{tabular}

aFluorescence emission intensity of solutions of $1 \mu \mathrm{M}$ PDI and $1 \mu \mathrm{M}$ DNA in 3:1 $25 \mathrm{mM}$ ammonium acetate/methanol buffer, $\mathrm{pH}$ 7, expressed as a percent of emission intensity for solutions of the PDI alone. ${ }^{\mathrm{b}}\left[\mathrm{d}\left(\mathrm{T}_{2} \mathrm{G}_{5} \mathrm{~T}\right)\right]_{4}$.

${ }^{\mathrm{C}}[\mathrm{d}(\mathrm{GCAAATTTCG})]_{2}$.

${ }^{\mathrm{d}}\left[\mathrm{d}\left(\mathrm{T}_{8}\right)\right]$.

excitation $=495 \mathrm{~nm}$, emission $=545 \mathrm{~nm}$.

${ }^{\mathrm{f}}$ Excitation $=495 \mathrm{~nm}$, emission $=555 \mathrm{~nm}$.

${ }^{\mathrm{g}}$ Excitation $=469 \mathrm{~nm}$,emission $=565 \mathrm{~nm}$.

The results of the fluorescence-quenching studies of the PDIs are summarized in Table 2, which shows the percent PDI fluorescence-quenching after the addition of one equivalent of the different DNA structures. In accord with the UV-Vis absorbance studies (Figure 2a), Tel01 displays a high degree of selectivity for G-quadruplex DNA binding, as shown by the minimal fluorescencequenching in the presence of either duplex or singlestranded DNA compared to G-quadruplex DNA.

Other PDIs, such as PIPER and Tel11, appear to interact more strongly with G-quadruplex DNA when compared to Tel01, but show very little selectivity for
G-quadruplex DNA versus duplex DNA. Both PIPER and Tel18 also show significant interactions with singlestranded DNA; the addition of one equivalent of singlestranded DNA to these PDIs quenches their fluorescence by more than 50\%. Tel18 and Tel34 appear to be of intermediate selectivity for G-quadruplex DNA versus duplex DNA and have moderate affinity for singlestranded DNA. Tel12 does not interact strongly with G-quadruplex DNA, as evidenced by only $29 \%$ fluorescence-quenching in the presence of one equivalent of G5-DNA; however, this PDI is relatively selective for G-quadruplex DNA and does not bind to singlestranded DNA. There was insignificant fluorescencequenching of Tel32, indicating that this PDI does not interact with any of these DNA structures.

\section{Binding Stoichiometry of Perylene Diimides by Electrospray Ionization Mass Spectrometry}

To begin an ESI-MS investigation of the binding selectivity of the perylene diimide ligands, the complexation of each ligand with G-quadruplex DNA was evaluated. The intermolecular, parallel stranded G-quadruplex $\left[\mathrm{d}\left(\mathrm{T}_{2} \mathrm{G}_{5} \mathrm{~T}\right)\right]_{4}(\mathrm{G} 5)$ was used in this study. Rosu et al. were the first to demonstrate that G-quadruplex DNA can be annealed in an ammonium acetate buffer and analyzed by ESI-MS [27]. When ammonium acetate is used, the G-quadruplex structure is stabilized by $\mathrm{NH}_{4}{ }^{+}$counterions, rather than by $\mathrm{Na}^{+}$or $\mathrm{K}^{+}$, resulting in cleaner spectra as a result of the greater lability of the associated counterions during the ESI process. The ESI mass spectrum of the G-quadruplex without any added ligands (Figure 3a) demonstrates that the G-quadruplex

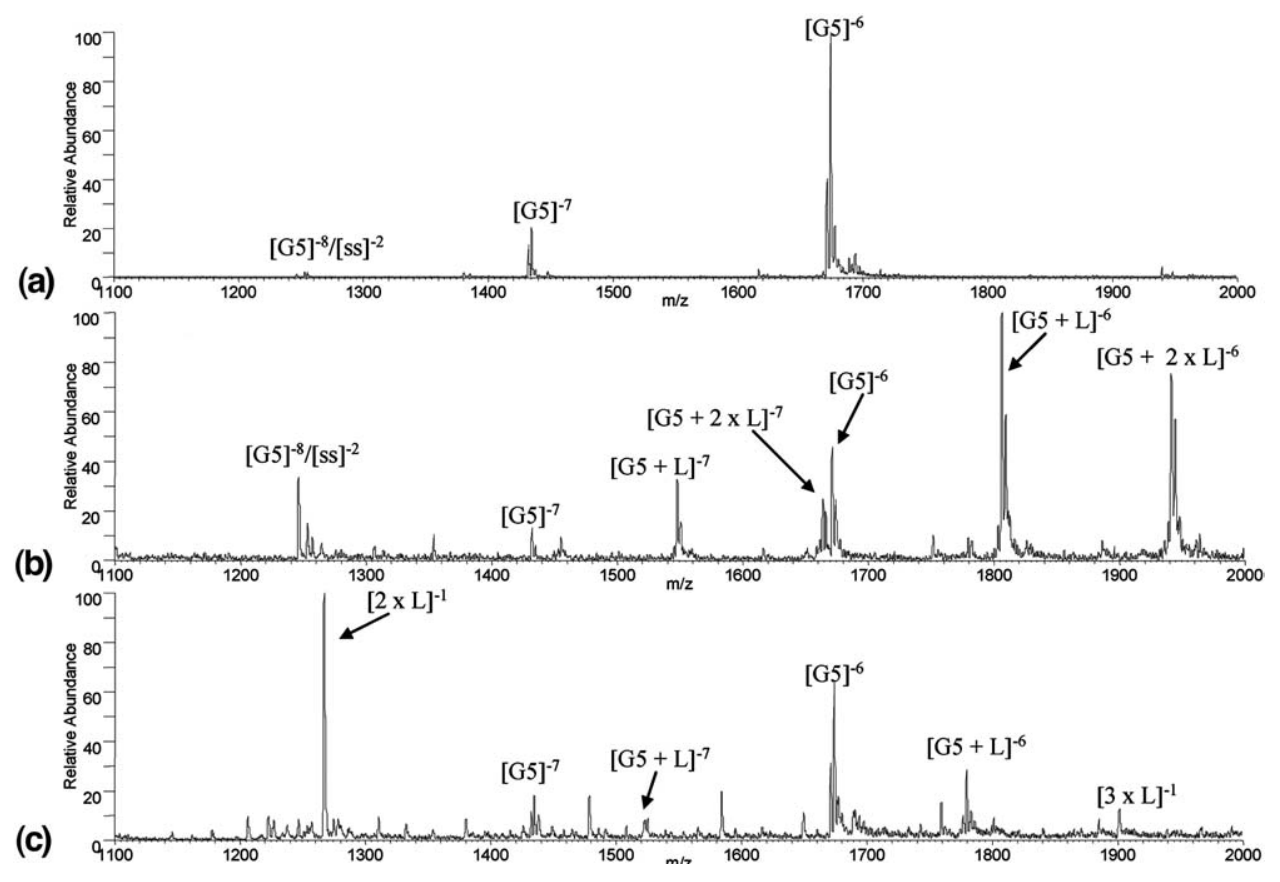

Figure 3. ESI mass spectra for complexes containing $\left[d\left(\mathrm{~T}_{2} \mathrm{G}_{5} \mathrm{~T}\right)\right]_{4}(\mathrm{G} 5)$ (a) alone, and with (b) Tel18 and (c) Tel12. Complexes containing one or more ligands are labeled with a "L". 
species can readily be detected in the -6 charge state, and to a lesser degree in the -7 charge state with two to four ammonium adducts associated with each quadruplex. Likely the ammonium ions are bound in the central cavity of the G-tetrad, stabilizing the quadruplex. For visual simplicity, the $\mathrm{NH}_{4}{ }^{+}$ions are not labeled in the remaining figures.

Solutions containing the G-quadruplex and one of each of the five non-benzannulated ligands (Tel01, Tel11, Tel12, Tel18, and PIPER) at equimolar concentrations of $10 \mu \mathrm{M}$ in a 3:1 $25 \mathrm{mM}$ ammonium acetate/ methanol solution exhibited a variety of binding stoichiometries as demonstrated by the mass spectra shown in Figure 3. For the solution containing Tel18 (Figure $3 b)$, complexes with Tel18/G5 binding stoichiometries of $1: 1$ and 2:1 are present with greater abundances than the free G-quadruplex ions. Other ligands formed less abundant complexes with the G-quadruplex. This is apparent in the spectrum of G5 with Tel12 (Figure 3c) in which only 1:1 complexes are present at lower abundances than the unbound G-quadruplex ions.

With the exception of Tel01 and Tel12, 1:1 and 2:1 binding stoichiometries were consistently observed. This result is in agreement with a previous NMR-based study of the perylene diimide analog PIPER that found 1:1 and 2:1 complexes were typically formed in solution by end-stacking on the faces of the terminal G-tetrads [35]. The 3:1 complexes present in the ESI mass spectrum of Tel01 with G5 may be the result of ligand aggregation, as discussed above. Recent X-ray crystallographic studies of an anthraquinone bound to a parallel-stranded G-quadruplex DNA show three ligands bound to a single G-tetrad face [45]. Presumably, a similar sort of self-association of multiple PDI ligands on G-tetrad faces could occur for Tel01, which undergoes aggregation in solution even in the absence of G-quadruplex DNA. Ligand aggregation is not observed in solution for the analogs with charged side chains (Tel11, Tel12, and Tel18). Aggregation is less expected for these ligands due to charge repulsion, which is especially true in the gas phase, thus explaining why aggregation was not observed with those ligands. Charge repulsion may also explain why only 1:1 complexes were observed for Tel12. As the only ligand with negatively charged side chains, Tel12 may experience coulombic repulsion with the anionic backbone of the DNA in solution and the gas-phase, preventing the binding of multiple ligands. In general, the predominant 1:1 and 2:1 binding stoichiometries observed for the ligands with G5 DNA is expected based on an end-stacking binding mode.

\section{Binding Stoichiometry of Benzannulated Ligands by ESI-MS}

The interactions of G-quadruplex DNA with a series of benzannulated perylene diimides (Figure 1) with an extended chromophore, Tel32 and Tel34, were also examined by ESI-MS. The benzannulated analogs may engage in increased end-stacking interactions with the G-tetrad as a result of the larger conjugated ring system The ESI-MS results for this set of analogs (spectra not shown) indicate that Tel34 forms complexes with the quadruplex DNA, but only with binding stoichiometries of 1:1. Interestingly, Tel11 and Tel18 have the same side-chain as Tel34, but both formed complexes with higher binding stoichiometries than Tel34, indicating that the benzannulated analogs may interact differently with the G-quadruplex. The larger chromophore of the benzannulated analogs may prevent multiple ligands from binding to the G5 structure due to steric hindrance. While Tel34 formed complexes with Gquadruplex DNA, the other benzannulated analog, Tel32, was the only perylene diimide ligand that did not form any complexes with G5 DNA.

\section{Concentration Effects}

To examine how changes in the solution conditions affect the binding stoichiometries of the ligand/G5 DNA complexes, solutions containing $10 \mu \mathrm{M}$ G5 DNA and either 2.5, 5.0, 10, and $20 \mu \mathrm{M}$ each PDI ligand were analyzed by ESI-MS (spectra not shown). The binding stoichiometries of the analogs containing charged side chains (Tel11, Tel12, Tel18, and Tel34), did not change from those observed at a 1:1 ligand/G5 ratio with either higher or lower ligand/G5 M ratios. For example, when the Tel12/G5 M ratio was 1:4, only complexes with a 1:1 binding stoichiometry were present in the ESI mass spectra. When the Tel12/G5 M ratio was increased from $1: 4$ to $2: 1$, the relative abundances of the $1: 1$ peaks remained the same and no 2:1 complexes appeared.

Ligands containing basic side chains (Tel01 and PIPER) do show concentration-dependent binding. At ligand/DNA molar ratios of 1:4 to 1:1, the complexes with binding stoichiometries of 1:1 are the most abundant, with 2:1 complexes present at relative abundances that are approximately three times less than that observed for the 1:1 complexes. When the ligand/DNA ratio is increased above 1:1, the 2:1 complexes become nearly twice as abundant as the 1:1 complexes, and very low abundance 3:1 complexes emerge that were not present at lower ligand/DNA ratios. The concentration dependent binding of Tel01 was demonstrated in a past study by David et al. [26], and is confirmed by experiments in this study. These results indicate that while all of the perylene diimides bind G-quadruplex DNA at primarily 1:1 and 2:1 binding stoichiometries, ligands with side chains that are basic, Tel01 and PIPER, demonstrate the ability to bind with higher stoichiometries at increased ligand/G5 M ratios. Both Tel01 and PIPER can undergo aggregation in solution in a $\mathrm{pH}-$ dependent fashion [37, 38]. The observation of higher binding stoichiometries for these two ligands may be due to ligand self-association during ESI, as supported by the RLS and fluorescence data discussed above and summarized in Table 1. 


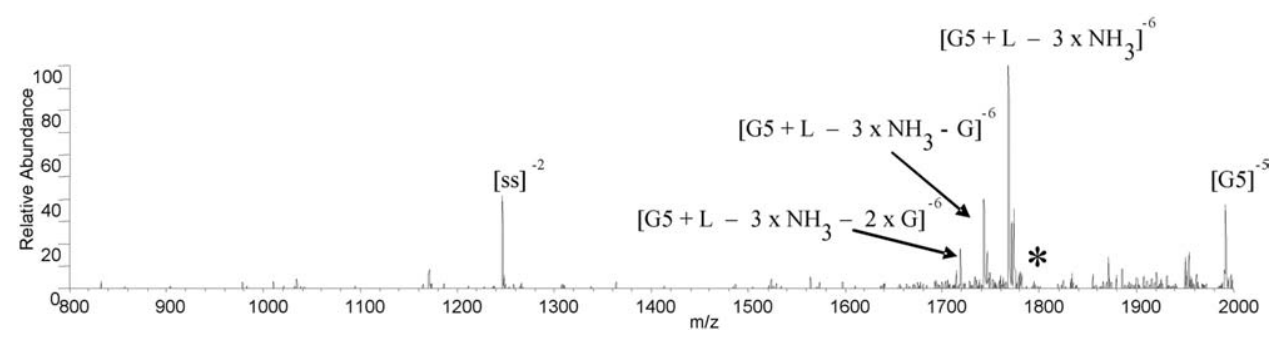

Figure 4. CAD product ion spectrum [G5 + Tel12] $]^{-6}$. Activation energy of $1.98 \mathrm{~V}$ was used. Complexes containing one or more ligands are labeled with an "L". Parent ions are labeled with an asterisk.

\section{ESI-MS/MS Studies of G5 DNA/Perylene Diimide Complexes}

ESI-MS/MS experiments were undertaken to examine the fragmentation patterns of ligand/G-quadruplex complexes via collisional activated dissociation. While full-scan ESI mass spectra are useful for comparing the binding stoichiometries of the various ligands with DNA, ligand binding modes are generally not distinguishable. Past ESI-MS/MS studies of drug/DNA complexes suggest that complexes with different binding modes produce distinct fragmentation patterns upon collisional activation $[6,7,26]$. In this study, all complexes, including $[\mathrm{G} 5+\mathrm{L}]^{-6},[\mathrm{G} 5+2 \times \mathrm{L}]^{-6},[\mathrm{G} 5+3$ $\times \mathrm{L}]^{-6},[\mathrm{G} 5+\mathrm{L}]^{-7}$, and $[\mathrm{G} 5+2 \times \mathrm{L}]^{-7}$, where $\mathrm{L}$ represents a perylene diimide ligand, were subjected to CAD experiments. All complexes, regardless of the charge of the ligand, the charge state of the complex, or the binding stoichiometry, dissociated via guanine base losses from the precursor ions in addition to the ejection of one anionic or neutral ligand, leaving the intact quadruplex. Triplex and single strand oligonucleotide ions without bound ligand are also present in the spectra as a result of further dissociation of the quadruplex. Ligands Tel12, Tel01, and PIPER were ejected as anions, while Tel11, Tel18, and Tel34 were ejected as neutral species. An example of this fragmentation pattern is shown in Figure 4 for the CAD spectrum of the $[\mathrm{G} 5+\text { Tel012 }]^{-6}$ complex. In addition to ions resulting from guanine base loss, the -5 charge state of the intact quadruplex and the -2 charge state of the single strand species are present. Presumably, the -3 charge state of the triplex is also formed; however the $\mathrm{m} / \mathrm{z}$ of this ion lies outside the detectable mass range of our instrument.

The facile loss of ligand observed in the CAD spectra of the DNA/perylene diimide complexes is consistent with past studies of ligands that bind to G-quadruplex DNA by end-stacking [26, 27] suggesting the perylene diimides bind to quadruplex DNA in a similar manner. Previously published results by our group demonstrated that complexes containing distamycin A and diethyloxadicarbocyanine (DTC), ligands that presumably bind to G-quadruplex DNA via groove binding $[46,47]$, produce single stranded, double stranded, and triplex ODNs with and without bound ligand upon
CAD [26]. It is important to note that both distamycin A and DTC are cationic, while the postulated quadruplex end-stacking ligands involved in a past ESI-MS/MS study [26] were neutral. It has been difficult to determine whether the differences between the fragmentation patterns of the end-stacking and the groovebinding quadruplex-interactive ligands were the result of different binding modes and/or ligand charge. However, in this study, all analogs, including the cationic Tel11, Tel18, and Tel34 ligands dissociated via the ejection of ligand from the intact G-quadruplex, suggesting that the charge of the ligand has less of an impact on the dissociation pathway than binding mode.

\section{Duplex and single strand DNA binding of ligands by ESI-MS}

The evaluation of duplex versus quadruplex binding selectivity is a critical issue in the design of new anti-cancer drugs. To evaluate selectivity, ESI mass spectra of solutions containing the perylene diimide ligands with duplex DNA (abbreviated as ds) have also been obtained to allow comparison of the selectivity of the ligands for quadruplex versus duplex versus single strand ODNs. To account for potential sequence selectivity, a series of three non-self-complementary duplex ODNs with varying G-C and A-T base pair composition were used (d(GCGGGGATGGGGCG/CGCCCCATCCCCGC), d(GCGGGAATTGGGCG/CGCCCAATTCCCGC), and d(GCGGAAATTTGGCG/CGCCAAATTTC(GC)). As shown in Figure 5, there is considerable variation in duplex versus quadruplex selectivity. Some ligands, such as Tel18, formed numerous complexes with the duplex DNA (Figure 5b), while others formed no complexes, as demonstrated by the spectrum containing Tel34 (Figure 5c). The increased number of adducts, especially for the single strand ions, present in Figure $5 c$ are due to higher levels of salts present in the Tel034 ligand sample. The ligands that did bind to the duplex DNA, namely PIPER, Tel11, and Tel18, formed complexes with binding stoichiometries of 4:1, 3:1, 2:1, and 1:1, and did not demonstrate any notable base pair selectivity.

To further examine the interaction between the ligands and duplex DNA, CAD experiments were per- 

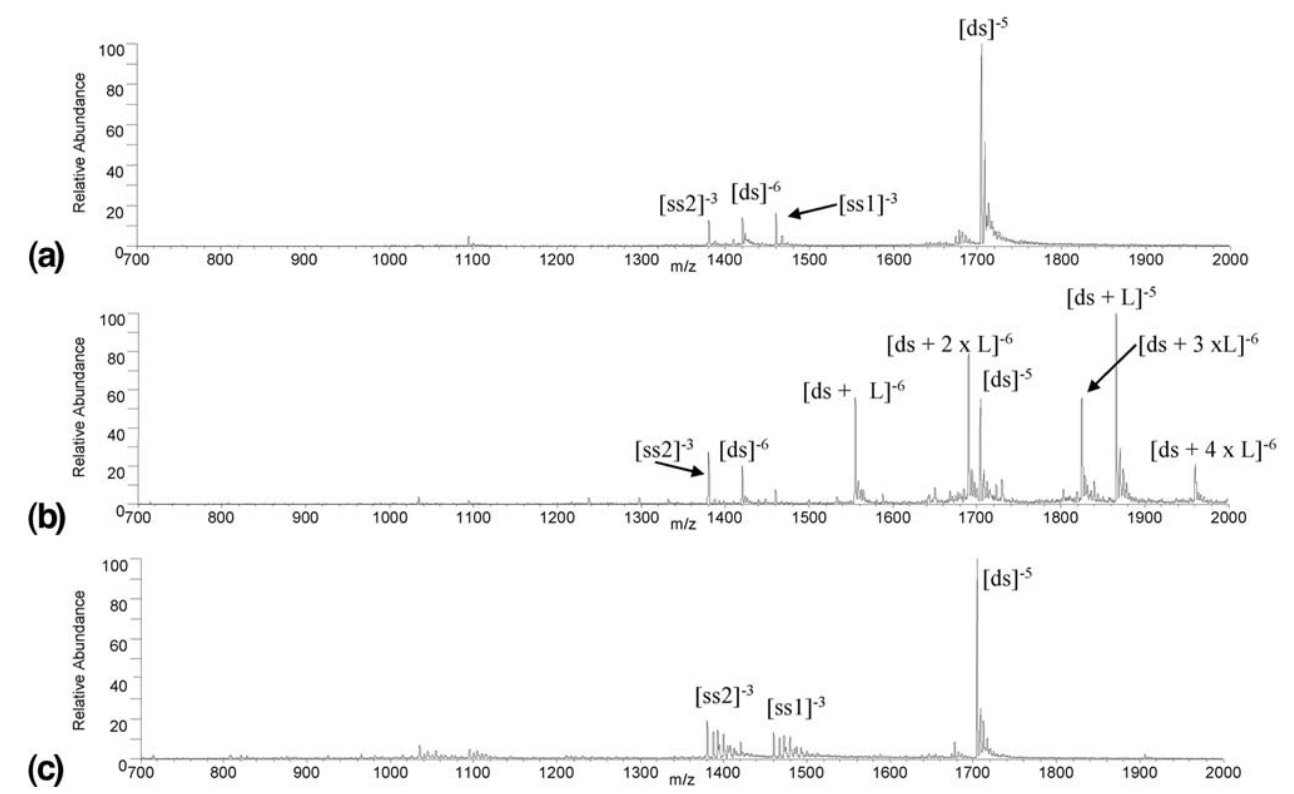

Figure 5. ESI mass spectra for complexes containing d(GCGGGAATTGGGCG/CGCCCAATTCCCGC): (a) alone, and with (b) Tel18 (extensive binding) and (c) Tel34 (no binding). The d(GCGGAATTGGGCG) single strand is labeled "ss1" and d(CGCCCAATTCCCGC) is labeled "ss2".

formed on all ligand/DNA complexes present in the mass spectra. The complexes subjected to CAD included $[\mathrm{ds}+\mathrm{L}]^{-5},[\mathrm{ds}+\mathrm{L}]^{-6},[\mathrm{ds}+2 \times \mathrm{L}]^{-6},[\mathrm{ds}+3$ $\times \mathrm{L}]^{-6},[\mathrm{ds}+4 \times \mathrm{L}]^{-6}$, where "L" represents either PIPER, Tel11, or Tel18. All complexes produced the same charge-state dependent CAD pattern, regardless of the ligand or binding stoichiometry. At lower charge states, such as -5 , the complexes dissociated by loss of nucleobases (specifically A, G, and C) from the intact complex, while at higher charge states, strand scission was observed, with one or more ligands remaining bound to the single strand ODN. An example of this characteristic fragmentation is shown in Supplementary Figure S-3 (which can be found in the electronice version of this article.) for the 1:1 complexes containing $\mathrm{d}$ (GCGGGAATTGGGCG/CGCCCAATTCCCGC) and Tel18.

The complexes containing PIPER, the only analog without cationic side chains that bound to duplex DNA, exhibited an additional fragmentation route. At low charge states, neutral ligand ejection was observed (a new route), leaving the duplex ion, in addition to base losses from the complex. These two processes are competitive since both types of fragments are formed from the same parent ion. The fragmentation pattern produced by the PDI/duplex DNA complexes, characterized by both noncovalent strand scission and ligand ejection, in addition to covalent cleavages of high proton affinity nucleobases from the complex, is similar to the results of past MS/MS studies of intercalator/ duplex DNA complexes [5, 7]. In quadrupole ion trap instruments, complexes containing minor groove binding ligands also undergo strand scission and cleavage of nucleobases, and covalent cleavages of the terminal portions of the duplex resulting in losses of $a_{n}$ and $\left(d_{n}\right.$ $+\mathrm{B}$ ) ions are also observed upon CAD [7]. The loss of these small sequence ions is never observed in the CAD spectra of the PDI/duplex complexes. Intercalation has been suggested for perylene diimide molecules that bind to duplex DNA [37, 38], and would be expected with a duplex DNA-interactive molecule containing a planar chromophore. Future work is necessary to examine the correlation between the fragmentation patterns of the perylene diimide/duplex complexes and ligand binding mode.

ESI mass spectra of solutions containing one ligand and a single strand ODN were also acquired. The single strand sequence used to anneal quadruplex DNA, $d\left(T_{2} G_{5} T\right)$, was used for these experiments to determine whether the analogs that selectively bound to the Gquadruplex DNA were selective for the G-quadruplex structure or for the sequence of DNA. To ensure that there were no Hoogsteen base pair interactions between the guanine residues of the single strand ODN, ESI mass spectra were first obtained for the ODN alone (Supplementary Material Figure S-4 which can be found in the electronic version of this article.). The mass spectrum shows that the most abundant ions are found at $\mathrm{m} / \mathrm{z} 831$ and 1247, corresponding to the [ss] ${ }^{-3}$ and $[\mathrm{ss}]^{-2}$ species, respectively. The appearance of this mass spectrum is significantly different than the one obtained for the annealed G-quadruplex (Figure 3a).

Solutions containing the single strand ODN and each ligand were prepared at equimolar $10 \mu \mathrm{M}$ concentration in 3:1 water/methanol. The ESI mass spectra show that the two ligands with cationic side chains that formed complexes with duplex DNA and quadruplex DNA, Tel11 and Tel18, also formed complexes with the 
Table 3. Summary of ligand binding selectivity ${ }^{a}$

\begin{tabular}{lcccccc}
\hline Ligand & PIPER & Tel01 & Tel11 & Tel12 & Tel18 & Tel34 \\
\hline \hline G5 Binding & 0.95 & 0.65 & 0.95 & 0.30 & 0.75 & 0.30 \\
Duplex Binding & 0.80 & 0.20 & 0.95 & - & 0.90 & -- \\
Single Strand Binding & -- & -- & 0.10 & -- & 0.10 & -- \\
Selectivity for G-Quadruplex & poor & good & poor & good & poor & good \\
\hline
\end{tabular}

"--" Indicates no complexes were observed. All values +/-0.05.

a $V$ alues in the chart represent the fraction of bound DNA.

single strand ODN (Figure S-4B), while the remaining ligands exhibited no single strand binding (Figure S-4C). These results suggest that Tel11 and Tel18 indiscriminately bind to DNA, regardless of the higher order structure. This lack of selectivity may be the result of electrostatic interactions between the cationic side chains of the analogs and the phosphate backbone of DNA. However, Tel34 also has a cationic side-chain but was only found to bind to G-quadruplex DNA, suggesting that the ligand/DNA complexes observed in the ESI mass spectra are not merely the result of nonspecific electrostatic interactions in the gas-phase.

\section{ESI-MS Evaluation of Binding Selectivity of Ligands}

After evaluating the binding of the ligands to Gquadruplex, duplex, and single strand DNA by ESIMS/MS, the overall selectivity of the analogs is summarized in Table 3. The values in the table represent the fraction of bound DNA calculated using the following equation:

Fraction of bound DNA

$$
=\frac{I_{(1: 1)}+I_{(2: 1)}+I_{(3: 1)}+I_{(4: 1)}}{I_{(\mathrm{DNA})}+I_{(1: 1)}+I_{(2: 1)}+I_{(3: 1)}+I_{(4: 1)}}
$$

where $I_{\mathrm{DNA}}$ is the relative abundance of the free (unbound DNA) and $I_{(\mathrm{n}: 1)}$ are the relative abundances of the ligand/DNA complexes. This equation is a modified version of an equation developed by Rosu et al. [17] that was used to estimate the concentration of all individual species at equilibrium based on the relative abundances of DNA ions in the mass spectrum, with the assumption that the abundances of the free and bound DNA ions are proportional to their relative concentrations in solution. We are not convinced that the ESI response factors of the unbound DNA ions and the DNA/perylene diimide complexes are identical, so instead we calculate the fraction of bound DNA to obtain a relative comparison of the extent of ligand binding to the different DNA structures.

While PIPER, Tel11, and Tel18 readily formed complexes with the quadruplex DNA, these three analogs also bound to duplex DNA which is undesirable for target selectivity. Both Tel11 and Tel18 were also found to bind to single strand DNA, suggesting that the cationic side chains of the ligands may promote indiscriminant binding to DNA due to electrostatic interactions with the anionic phosphate backbone. Interestingly, Tel34 has a cationic side-chain but did not bind to duplex or single strand DNA, thus exhibiting significant selectivity. This suggests the side-chains of the benzannulated analogs have a different role in DNA binding, which may be a key issue for G-quadruplex selectivity.

The only analog with anionic side chains, Tel12, formed 1:1 complexes with the G5 DNA, but did not bind to duplex or single strand DNA. Whether the anionic side chains play a role in the selectivity of the analog is still unclear, but the binding of the ligand to the G-quadruplex suggests that the complexes observed in the ESI mass spectra are not solely the result of electrostatic interactions. Finally, PIPER was found to bind to G-quadruplex and duplex DNA, while the structurally-similar ligand, Tel01, demonstrated selectivity for the G-quadruplex.

There is good correlation between these ESI-MS results and the spectroscopic studies of G-quadruplex DNA binding by these PDIs (see above). Both the UV-Vis spectroscopic studies and ESI-MS analysis demonstrate that all of the PDIs examined here, with the exception of Tel32, bind to parallel-stranded G-quadruplex DNA. Spectroscopic and ESI-MS studies also both reveal the selectivity of certain PDIs (e.g., Tel01) for G-quadruplex versus duplex DNA and the lack of selectivity of other PDIs (e.g., Tel11) for any of the DNA structures studied here. Additional insights into the origin of the G-quadruplex binding selectivity observed for certain PDIs is provided by ESI-MS. Of the PDIs studied, three were found to undergo extensive selfassociation (Tel12, Tel34) or aggregation (Tel01) in solution. All three of these ligands display selectivity for G-quadruplex DNA. However, ESI-MS reveals that the stoichiometries of G-quadruplex DNA binding by these ligands are different. Tel01 binds to G-quadruplex DNA to form complexes with as many as three ligands associated with a single quadruplex. Tel12 and Tel34 only form 1:1 complexes with G-quadruplex DNA. Nonselective PDIs such as PIPER and Tel11, which are not aggregated or highly self-associated in solution, also form 1:1 and 2:1 ligand/DNA complexes with G-quadruplex DNA. Clearly, the selectivity of PDIs for G-quadruplex DNA binding is correlated with the 
self-association state of these ligands in solution and not their self-association on G-quadruplex DNA.

\section{Conclusions}

The results of this study demonstrate the use of ESI-MS to evaluate noncovalent interactions between perylene diimide ligands and G-quadruplex DNA and to screen the ligands for selectivity. There is good correlation between spectroscopic binding studies and ESI-MS analysis of G-quadruplex DNA binding. Qualitative comparisons can be made to quickly screen ligands with minimal consumption of analyte, making this technique compatible with high throughput screening techniques. Our results found, with the exception of Tel32 that exhibited no DNA binding, all ligands evaluated in this study formed complexes with Gquadruplex DNA. Binding stoichiometries of 1:1 and 2:1 were observed for PIPER, Tel11, and Tel18; this is a binding stoichiometry that is consistent with telomerase inhibitors that bind to G-quadruplex DNA by stacking on the faces of the terminal G-tetrads [35, 40]. Interestingly, the three most quadruplex-selective ligands formed complexes with quadruplex DNA with a different binding stoichiometry. Tel01 formed 3:1 complexes, and Tel12 and Tel18 only formed 1:1 complexes. This unusual behavior will be explored in future studies. CAD experiments also suggested an end-stack mode of binding since all complexes dissociated by ejecting the ligand, leaving the intact G-quadruplex, triplex, or single strand ODN. Based on binding to G-quadruplex, duplex, and single stranded DNA, the analogs Tel01, Tel12, and Tel34 are most promising in terms of Gquadruplex selectivity. Our future work will focus on obtaining more quantitative information about ligand/ G-quadruplex complexes, such as binding constants, and identification of specific binding sites.

\section{Acknowledgments}

Funding from the Robert A. Welch Foundation (F-1155 to JSB and F-1298 to SMK) and the National Institutes of Health (RO1 GM65956) is gratefully acknowledged.

\section{References}

1. Goodman, L. S.; Hardman, J. G.; Limbird, L. E.; Gilman, A. G. Goodman and Gilman's The Pharmacological Basis of Therapeutics, 10th ed.; McGrawHill: New York, 2001, pp 1381-1460.

2. Propst, C. L.; Perun, T. J. Nucleic Acid Targeted Drug Design; Marcel Dekker: New York, 1992, pp 1-12.

3. Hofstadler, S. A.; Griffey, R. H. Analysis of Noncovalent Complexes of DNA and RNA by Mass Spectrometry. Chem. Rev. 2001, 101, 377-390.

4. Beck, J. L.; Colgrave, M. L.; Ralph, S. F.; Sheil, M. M. Electrospray Ionization Mass Spectrometry of Oligonucleotide Complexes with Drugs, Metals, and Proteins. Mass Spectrom. Rev. 2001, 20, 61-87.

5. Gabelica, V.; De Pauw, E. Comparison of the Collision-Induced Dissociation of Duplex DNA at Different Collision Regimes: Evidence for a Multistep Dissociation Mechanism. J. Am. Soc. Mass Spectrom. 2001, 13, 91-98.

6. Gabelica, V.; De Pauw, E.; Rosu, F. Interaction Between Antitumor Drugs and a Double Stranded Oligonucleotide Studied by Electrospray Ionization Mass Spectrometry. J. Mass Spectrom. 1999, 34, 1328-1337.

7. Wan, K. X.; Gross, M. L.; Shibue, T. Gas-Phase Stability of DoubleStranded Oligodeoxynucleotides and Their Noncovalent Complexes with DNA-Binding Drugs Revealed by Collisional Activation in an Ion Trap. J. Am. Soc. Mass Spectrom. 2000, 11, 450-457.

8. Gale, D. C.; Smith, R. D. Characterization of Noncovalent Complexes Formed Between Minor Groove Binding Molecules and Duplex DNA by Electrospray Ionization Mass Spectrometry. J. Am. Soc. Mass Spectrom. 1995, 6, 1154-1164.

9. Triolo, A.; Arcamone, F. M.; Raffaelli, A.; Salvadori, P. Noncovalent Complexes Between DNA-Binding Drugs and Doubly Stranded Deoxyoligonucleotides: A Study by Ion Spray Mass Spectrometry. J. Mass Spectrom. 1997, 32, 1186-1194.

10. Hofstadler, S. A.; Sannes-Lowery, K. A.; Crooke, S. T.; Ecker, D. J.; Sasmor, H.; Manalili, S.; Griffey, R. H. Multiplexed Screening of Neutral Mass-Tagged RNA Targets Against Ligand Libraries with Electrospray Ionization FTICR MS: A Paradigm for High-Throughput Affinity Screening. Anal. Chem. 1999, 71, 3436-3440.

11. Kapur, A.; Beck, J. L.; Sheil, M. M. Observation of Daunomycin and Nogalamycin Complexes with Duplex DNA Using Electrospray Ionization Mass Spectrometry. Rapid Commun. Mass Spectrom. 1999, 13, $2489-$ 2497.

12. Greig, M. J.; Robinson, J. M. Detection of Oligonucleotide-Ligand Complexe by ESI-MS (DOLCE-MS) as a Component of High Throughput Screening. J. Biomol. Screen. 2000, 5, 441-454.

13. Wan, K. X.; Shibue, T.; Gross, M. L. Noncovalent Complexes Between DNA-Binding Drugs and Double Stranded Oligodeoxynucleotides: A Study by ESI Ion Trap Mass Spectrometry. J. Am. Chem. Soc. 2000, 122, 300-307.

14. Gabelica, V.; Rosu, F.; Houssier, C.: De Pauw, E. Gas Phase Thermal Denaturation of an Oligonucleotide Duplex and Its Complexes with Minor Groove Binders. Rapid Commun. Mass Spectrom. 2000, 14, 464467.

15. Iannitti-Tito, P.; Weimann, A.; Wickham, G.; Sheil, M. M. Structural Analysis of Drug-DNA Adducts by Tandem Mass Spectrometry. Analyst 2000, 125, 627-633.

16. Gupta, R.; Kapur, A.; Beck, J. L.; Sheil, M. M. Positive Ion Electrospray Ionization Mass Spectrometry of Double Stranded DNA/Drug Complexes. Rapid Commun. Mass Spectrom. 2001, 15, 2472-2480.

17. Rosu, F.; Gabelica, V.; Houssier, C.; De Pauw, E. Determination of Affinity, Stoichiometry, and Sequence Selectivity of Minor Groove Complexes with Double Stranded Oligodeoxynucleotides by Electrospray Ionization Mass Spectrometry. Nucleic Acids Res. 2002, 30, e82.

18. Carrasco, C.; Rosu, F.; Gabelica, V.; Houssier, C.; De Pauw, E.; GarbayJaureguiberry, C.; Roques, B.; Wilson, W. D.; Chaires, J. B.; Waring, M. J.; Bailly, C. Tight Binding of the Anticancer Drug Ditercalinium to Quadruplex DNA. Chem. Biochem. 2002, 3, 1235-1241.

19. Colgrave, M. L.; Beck, J. L.; Sheil, M. M.; Searle, M. S. Electrospray Ionization Mass Spectrometric Detection of Weak Noncovalent Interactions in Nogalamycin-DNA Complexes. Chem. Commun. 2002, (6), $556-557$.

20. Guittat, L.; Alberti, P.; Rosu, F.; Van Miert, S.; Thetiot, E.; Pieters, L.; Gabelica, V.; De Pauw, E.; Ottaviani, A.; Riou, J. F.; Mergny, J. L. Interactions of Cryptolepine and Neocrptolepine with Unusual DNA Structures. Biochimie, 85, 535, 2003, 547.

21. Gabelica, V.; Galic, N.; Rosu, F.; Houssier, C.; De Pauw, E. Influence of Response Factors on Determining Equilibrium Association Constants of Noncovalent Complexes by Electrospray Ionization Mass Spectrometry. J. Mass. Spectrom. 2003, 38, 491-501.

22. Colgrave, M. L.; Iannitti-Tito, P.; Wickham, G.; Sheil, M. M. Rapid Determination of Sequence Selectivity and Stability of Alkylated Oligonucleotide Adducts by Electrospray Tandem Mass Spectrometry. Aust. J. Chem. 2003, 56, 401-413.

23. Rosu, F.; De Pauw, E.; Guittat, L.; Alberti, P.; Lacroix, L.; Mailliet, P.; Riou, J. F.; Mergny, J. L. Selective Interaction of Ethidium Derivatives with Quadruplexes: An Equilibrium Dialysis and Electrospray Ionization Mass Spectrometry Analysis. Biochemistry 2003, 42, 10361-10371.

24. Rosu, F.; Gabelica, V.; Shin-ya, K.; De Pauw, E. Telomestatin-Induced Stabilization of the Human Telomeric DNA Quadruplex Monitored by Electrospray Mass Spectrometry. Chem. Commun. 2003, (21), 2702-2703.

25. Beck, J. L.; Gupta, R.; Urathamakul, T.; Williamson, N. L.; Sheil, M. M.; Aldrich-Wright, J. R.; Ralph, S. F. Probing DNA Selectivity of Ruthenium Metallointercalators using ESI Mass Spectrometry. Chem. Commun. 2003, (5), 626-627.

26. David, W. M.; Brodbelt, J.; Kerwin, S. M.; Thomas, P. W. Investigation of Quadruplex Oligonucleotide-Drug Interactions by Electrospray Ionization-Mass Spectrometry. Anal. Chem. 2002, 74, 2029-2033.

27. Rosu, F.; Gabelica, V.; Houssier, C.; Colson, P.; Pauw, E. D. Triplex and Quadruplex DNA Structures Studied by Electrospray Mass Spectrometry. Rapid Commun. Mass Spectrom. 2002, 16, 1729-1736.

28. Vairamani, M.; Gross, M. L. G-Quadruplex Formation of ThrombinBinding Aptamer Detected by Electrospray Ionization Mass Spectrometry. J. Am. Chem. Soc. 2003, 125, 42-43.

29. Kerwin, S. M. G-Quadruplex DNA as a Target for Drug Design. Curr. Pharm. Des. 2000, 6, 441-471.

30. Hurley, L. H.; Wheelhouse, R. T.; Sun, D.; Kerwin, S. M.; Salazar, M.; Fedoroff, O. Y.; Han, F. X.; Han, H. Y.; Izbicka, E.; Von Hoff, D. D. G-Quadruplexes as Targets for Drug Design. Pharmacol. Ther. 2000, 85, 141-158.

31. Wellinger, R. J.; Sen, D. The DNA Structures at the Ends of Eukaryotic Chromosomes. Eur. J. Cancer 1997, 33, 735-749. 
32. Bearss, D. J.; Hurley, L. H.; Von Hoff, D. D. Telomere Maintenance Mechanisms as a Target for Drug Development. Oncogene 2000, 19, 6632-6641.

33. Mergny, J. L.; Mailliet, P.; Lavelle, F.; Riou, J. F.; Laoui, A.; Helene, C. The Development of Telomerase Inhibitors: The G-Quartet Approach. Anti-Cancer Drug Des. 1999, 14, 327-339.

34. Counter, C. M.; Hahn, W. C.; Wei, W. Y.; Caddle, S. D.; Beijersbergen, R. L.; Lansdorp, P. M.; Sedivy, J. M.; Weinberg, R. A. Dissociation Among in Vitro Telomerase Activity, Telomere Maintenance, and Cellular Immortalization. Proc. Natl. Acad. Sci. U.S.A. 1998, 95, 1472314728 .

35. Fedoroff, O. Y.; Salazar, M.; Han, H. Y.; Chemeris, V. V.; Kerwin, S. M.; Hurley, L. H. NMR-Based Model of a Telomerase-Inhibiting Compound Bound to G-Quadruplex DNA. Biochemistry 1998, 37, 12367-12374.

36. Kern, J. T.; Kerwin, S. M. The Aggregation and G-Quadruplex DNA Selectivity of Charged 3,4,9,10-Perylenetetracarboxylic Acid Diimides. Bioorg. Med. Chem. Lett. 2002, 12, 3395-3398.

37. Kern, J. T.; Thomas, P. W.; Kerwin, S. M. The Relationship Between Ligand Aggregation and G-Quadruplex DNA Selectivity in a Series of 3,4,9,10-Perylenetetracarboxylic Acid Diimides. Biochemistry 2002, 41, $11379-11389$

38. Kerwin, S. M.; Chen, G.; Kern, J. T.; Thomas, P. W. Perylene Diimide G-Quadruplex DNA Binding Selectivity is Mediated by Ligand Aggregation. Bioorg. Med. Chem. Lett. 2002, 12, 447-450.

39. Rossetti, L.; Franceschin, M.; Bianco, A.; Ortaggi, G.; Savino, M. Perylene Diimides with Different Side Chains are Selective in Inducing
Different G-Quadruplex DNA Structures and in Inhibiting Telomerase. Bioorg. Med. Chem. Lett. 2002, 12, 2527-2533.

40. Read, M. A.; Neidle, S. Structural Characterization of a GuanineQuadruplex Ligand Complex. Biochemistry 2000, 39, 13422-13432.

41. Han, H. Y.; Bennett, R. J.; Hurley, L. H. Inhibition of Unwinding of G-Quadruplex Structures by Sgs1 Helicase in the Presence of N, $\mathrm{N}^{\prime}$-bis[2-(1-Piperidino)Ethyl]-3,4,9,10-Perylenetetracarboxylic Diimide, a G-Quadruplex-Interactive Ligand. Biochemistry 2000, 39, 9311-9316.

42. Han, H. Y., Cliff, C. L.; Hurley, L. H. Accelerated Assembly of G-Quadruplex Structures by a Small Molecule. Biochemistry 1999, 38, 6981-6986.

43. Read, M. A.; Wood, A. A.; Harrison, J. R.; Gowan, S. M.; Kelland, L. R.; Dosanjh, H. S.; Neidle, S. Molecular Modeling Studies on G-Quadruplex Complexes of Telomerase Inhibitors: Structure-Activity Relationships. J. Med. Chem. 1999, 42, 4538-4546.

44. Pasternack, R. F.; Collings, P. J. Resonance Light-Scattering-A New Technique for Studying Chromophore Aggregation. Science 1995, 269, 935-939.

45. Clark, G. R.; Pytel, P. D.; Squire, C. J.; Neile, S. Structure of the First Parallel DNA Quadruplex-Drug Complex. J. Am. Chem. Soc. 2003, 125, 4066-4067.

46. Randazzo, A., Galeone, A.; Mayol, L. H-1-NMR Study of the Interaction of Distamycin A and Netropsin with the Parallel Stranded Tetraplex [d(TGGGGT)](4). Chem. Commun. 2001, (11), 1030-1031.

47. Kerwin, S. M.; Sun, D.; Kern, J. T.; Rangan, A.; Thomas, P. W. G-Quadruplex DNA Binding by a Series of Carbocyanine Dyes. Bioorg. Med. Chem. Lett. 2001, 11, 2411-2414. 